\begin{tabular}{|c|l|}
\hline Title & On the asy mptotic behavior of nonlinear waves in the presence of a short-range potential \\
\hline Author(s) & Karageorgis, Paschalis; Tsutaya, Kimitoshi \\
\hline Citation & Hokkaido University Preprint Series in Mathematics, 770, 1-20 \\
\hline Issue Date & 2006 \\
\hline DOI & 10.14943/83920 \\
\hline Doc URL & http://hdl.handle.net/2115/69578 \\
\hline Type & bulletin (article) \\
\hline File Information & pre770.pdf \\
\hline
\end{tabular}

Instructions for use 


\title{
ON THE ASYMPTOTIC BEHAVIOR OF NONLINEAR WAVES IN THE PRESENCE OF A SHORT-RANGE POTENTIAL
}

\author{
PASCHALIS KARAGEORGIS AND KIMITOSHI TSUTAYA
}

\begin{abstract}
Consider the nonlinear wave equation with zero mass and a time-independent potential in three space dimensions. When it comes to the associated Cauchy problem, it is already known that short-range potentials do not affect the existence of small-amplitude solutions. In this paper, we focus on the associated scattering problem and we show that the situation is quite different there. In particular, we show that even arbitrarily small and rapidly decaying potentials may affect the asymptotic behavior of solutions.
\end{abstract}

\section{INTRODUCTION}

Consider the nonlinear wave equation with potential

$$
\partial_{t}^{2} u-\Delta u+V(x) \cdot u=F(u) \quad \text { in } \mathbb{R}^{3} \times \mathbb{R},
$$

where $F(u)$ behaves like $|u|^{p}$ for some $p>1$. If the potential $V(x)$ is sufficiently small and rapidly decaying, then one might expect solutions to (NWP) to behave like solutions to

$$
\partial_{t}^{2} u-\Delta u=F(u) \quad \text { in } \mathbb{R}^{3} \times \mathbb{R} .
$$

When it comes to the associated Cauchy problems, for instance, the known sharp existence results for (NW) coincide with those for (NWP), if the potential is sufficiently short-range. In this paper, we focus on the associated scattering problems and we show that the situation is quite different there. More precisely, we show that the sharp conditions needed to define the scattering operator for (NW) are strictly weaker than those for (NWP), if the potential is non-positive but not identically zero. In particular, even short-range potentials may affect the asymptotic behavior of solutions.

Let us first focus on the associated Cauchy problems and prescribe initial data

$$
u(x, 0)=\varphi(x), \quad \partial_{t} u(x, 0)=\psi(x) .
$$

Sharp existence results for (NW) go back to the classical work of John [6], where $\varphi, \psi$ are assumed to have compact support. An extension of John's result to more general data was obtained by Asakura [1] and then slightly refined by Kubota [9] and Tsutaya [16]. In these results, one assumes that

$$
\sum_{|\alpha| \leq 3}\left|\partial_{x}^{\alpha} \varphi(x)\right|+\sum_{|\beta| \leq 2}\left|\partial_{x}^{\beta} \psi(x)\right| \leq \varepsilon(1+|x|)^{-k-1}
$$

for some $k>0$ and some small $\varepsilon>0$. To ensure the existence of classical solutions to the Cauchy problem associated with (NW), it then suffices to require that

$$
p>1+\sqrt{2}, \quad k \geq 2 /(p-1) .
$$

1991 Mathematics Subject Classification. 35L05; 35L70; 35P25.

Key words and phrases. Wave equation; Potential; Scattering. 
Recall that $p$ denotes the order of the nonlinear term. Conditions (1.3) are also known to be necessary for the existence of small-amplitude solutions. That is, there exist arbitrarily small initial data satisfying (1.2) for which the solution to (NW) blows up in finite time, if either $1<p \leq 1+\sqrt{2}$ or else $0<k<2 /(p-1)$ for some $p>1$; see $[1,4,6,14]$.

When it comes to the Cauchy problem associated with (NWP), the above results apply verbatim for all sufficiently short-range potentials. If either of the conditions (1.3) fails to hold, then one can still have blow up for arbitrarily small data and short-range potentials; see [7, 17]. And if conditions (1.3) hold, then a result of Strauss and Tsutaya [15] ensures the existence of classical solutions, provided that

$$
\sum_{|\alpha| \leq 2}\left|\partial_{x}^{\alpha} V(x)\right| \leq V_{0}(1+|x|)^{-2-\delta}, \quad \delta>0
$$

for some small $V_{0}>0$. Moreover, it was also shown in [15] that both the smallness and the decay assumption (1.4) are sharp, if the potential is assumed to be non-positive. Namely, a non-positive potential which is either large or decaying like $|x|^{-2+\delta}$ at infinity will force solutions to blow up in finite time, even in the case that conditions (1.3) hold; see also [7]. For non-negative potentials, on the other hand, the smallness assumption does not seem to be needed; this was illustrated in [2] for potentials of compact support.

Next, we turn to the associated scattering problems for initial data satisfying (1.2). When it comes to (NW), the existence of the scattering operator was shown by Pecher [13] under the assumptions

$$
1+\sqrt{2}<p \leq 3, \quad k>2 /(p-1) .
$$

An extension of this result to (NWP) was obtained by the first author [8] under the slightly different assumptions

$$
1+\sqrt{2}<p<3, \quad k \geq 2 /(p-1)
$$

for all short-range potentials satisfying (1.4). To interpret these results, it is perhaps better to think in terms of the energy norm

$$
\|u\|_{e}=\left(\int_{\mathbb{R}^{3}}\left|\partial_{t} u\right|^{2} d x+\int_{\mathbb{R}^{3}}|\nabla u|^{2} d x\right)^{1 / 2} .
$$

As one might expect from the assumption (1.2) on the initial data, the solutions constructed in $[8,13]$ behave like $|x|^{-k}$ at infinity. Thus, their first-order derivatives are in $L^{2}\left(\mathbb{R}^{3}\right)$ if and only if $k>1 / 2$. This also gives the range of decay rates $k$ for which finite-energy solutions arise. The results of $[8,13]$, on the other hand, are restricted to decay rates $k>1$ because of either (1.5) or (1.6). Thus, there exist finite-energy solutions to both (NW) and (NWP) whose asymptotic behavior has not been studied yet.

Our main goal in this paper is to establish the sharp conditions needed to define the scattering operator for both (NW) and (NWP) when the assumption (1.2) is imposed on the initial data. There are related results which impose more general assumptions, avoiding the pointwise estimate (1.2). Nevertheless, these results are not optimal with respect to the order of the nonlinearity; see $[5,11]$ and the references cited therein.

Let us denote by $u_{0}^{-}$the solution to the homogeneous wave equation

$$
\partial_{t}^{2} u_{0}-\Delta u_{0}=0 \quad \text { in } \mathbb{R}^{3} \times \mathbb{R}
$$


subject to the initial data (1.1). As it is well-known, one can obtain a solution $u$ to (NWP) by solving the associated integral equation

$$
u=u_{0}^{-}+\mathscr{L} F(u)-\mathscr{L}(V u)
$$

where the Duhamel operator $\mathscr{L}$ is defined by the formula

$$
[\mathscr{L} G](x, t)=\frac{1}{4 \pi} \int_{-\infty}^{t} \frac{1}{t-\tau} \int_{|y-x|=t-\tau} G(y, \tau) d S_{y} d \tau .
$$

Regarding the existence of solutions to (1.9), we shall prove the following

Theorem 1.1 (Existence). Let $u_{0}^{-}$be the solution to the homogeneous wave equation

$$
\partial_{t}^{2} u_{0}-\Delta u_{0}=0, \quad u_{0}(x, 0)=\varphi(x), \quad \partial_{t} u_{0}(x, 0)=\psi(x) .
$$

Assume (1.2), (1.3), (1.4) and take $F(u)=|u|^{p}$ or $F(u)=|u|^{p-1} u$. If $\varepsilon, V_{0}$ are sufficiently small, then the integral equation (1.9) has a unique $\mathcal{C}^{2}$-solution.

Remark 1.2. The solutions we construct lie in a certain Banach space that we introduce in the next section; see (2.4). We can similarly construct solutions for more general nonlinear terms than the ones listed here; our precise assumptions on $F$ appear in (2.8), (2.9).

The proof of Theorem 1.1 is essentially based on the work of Pecher [13] as well as other results for the Cauchy problem $[1,6,15,16]$. However, our assumptions on the initial data are more general than those in [13], while the domain of dependence does no longer extend over a bounded region. To establish the existence of solutions, we thus need to derive a new estimate for the inhomogeneous wave equation with a nonlinear term and a potential; see Lemma 3.1. For the homogeneous wave equation (1.8), we use the weighted $L^{\infty}$-estimates of Lemma 2.1, which are pretty well-known by now.

Regarding the existence of the scattering operator, we shall prove the following

Theorem 1.3 (Scattering). Let the assumptions of Theorem 1.1 hold. Also, assume

$$
\begin{array}{lll}
k p \geq k+2, & k>1 & \text { for the case } V(x) \neq \equiv ; \\
k p \geq k+2, & k p>5 / 2 & \text { for the case } V(x) \equiv 0 .
\end{array}
$$

Then the unique solution u provided by Theorem 1.1 satisfies

$$
\left\|u-u_{0}^{-}\right\|_{e} \rightarrow 0 \quad \text { as } t \rightarrow-\infty,
$$

and there exists a unique solution $u_{0}^{+}$to the homogeneous equation (1.8) which satisfies

$$
\left\|u-u_{0}^{+}\right\|_{e} \rightarrow 0 \quad \text { as } t \rightarrow+\infty \text {. }
$$

In particular, one can define the scattering operator $S: u_{0}^{-} \rightarrow u_{0}^{+}$.

Remark 1.4. The first condition in (1.11), (1.12) simply repeats one of our assumptions in Theorem 1.1. It is one of the necessary conditions (1.3) for the existence of solutions. Here, we have chosen to restate it explicitly in order to illustrate that the assumptions imposed in the presence of a short-range potential (1.11) are strictly stronger than those imposed in its absence (1.12). This fact only becomes clear when the first condition is included. 
Remark 1.5. Consider our finite-energy solutions, i.e., the ones with decay rates $k>1 / 2$. We assume the first condition in (1.12) to ensure their existence. Then the second condition follows automatically. In particular, our theorem shows that all our finite-energy solutions are asymptotically free in the absence of the potential. For short-range potentials, we have the same conclusion, yet only if $k>1$; this excludes some of our finite-energy solutions.

To complement our scattering result, Theorem 1.3, we shall also show that the conditions given there are actually sharp. More precisely, we shall also establish the following

Theorem 1.6 (No scattering). Let the assumptions of Theorem 1.1 hold. Also, assume either

$$
k p \geq k+2, \quad k \leq 1, \quad 0 \not \equiv(x) \leq 0
$$

or else

$$
k p \geq k+2, \quad k p \leq 5 / 2, \quad V(x) \equiv 0 .
$$

If the initial data are chosen so that

$$
\varphi(x) \equiv 0, \quad \psi(x) \leq 0, \quad|\psi(x)| \geq \varepsilon_{1}(1+|x|)^{-k-1}
$$

for some small $\varepsilon_{1}>0$, then the solution u provided by Theorem 1.1 does not satisfy (1.13).

Remark 1.7. The smallness assumption on $\varepsilon_{1}$ is merely needed to ensure the existence of solutions, as Theorem 1.1 is only applicable for small initial data. For large initial data, on the other hand, solutions to (NWP) need not even exist for all times; see [10].

Results analogous to Theorem 1.6 have been obtained by Glassey [3] and Matsumura [12] in the context of the nonlinear Klein-Gordon equation. However, their approach cannot be applied here because our solutions are not necessarily in $L^{2}$. To briefly outline our method of proof, our goal is to argue by contradiction. Assuming that (1.13) does hold, we are able to find a function $G(x, t)$ such that $G(\cdot, t) \in L^{2}\left(\mathbb{R}^{3}\right)$ for all times, whereas

$$
\int_{\mathbb{R}^{3}} G(x, t) \cdot \partial_{t}\left(u-u_{0}^{-}\right) d x
$$

fails to approach zero as $t \rightarrow-\infty$. By Hölder's inequality then, one cannot really have

$$
\left\|\partial_{t}\left(u-u_{0}^{-}\right)\right\|_{L^{2}\left(\mathbb{R}^{3}\right)} \rightarrow 0 \quad \text { as } t \rightarrow-\infty,
$$

so the convergence (1.13) in the energy norm has to fail as well.

The remaining of this paper is organized as follows. In section 2, we give the well-known weighted $L^{\infty}$-estimates for the homogeneous wave equation and then establish some useful estimates involving our weight function (2.6). In section 3, we combine these facts to prove our existence result, Theorem 1.1, and our scattering result, Theorem 1.3. Finally, the proof of Theorem 1.6 is given in section 4 .

\section{A PRIORI ESTIMATES}

In this section, we gather some estimates that will be needed in the proof of our existence result, Theorem 1.1. Let us first focus on the homogeneous wave equation

$$
\partial_{t}^{2} u_{0}-\Delta u_{0}=0 \quad \text { in } \mathbb{R}^{3} \times \mathbb{R}
$$

and impose the conditions

$$
u_{0}(x, 0)=\varphi(x), \quad \partial_{t} u_{0}(x, 0)=\psi(x) .
$$


When it comes to the initial data, we shall assume that

$$
\sum_{|\alpha| \leq 3}\left|\partial_{x}^{\alpha} \varphi(x)\right|+\sum_{|\beta| \leq 2}\left|\partial_{x}^{\beta} \psi(x)\right| \leq \varepsilon\langle x\rangle^{-k-1},
$$

where $\langle x\rangle=1+|x|$ and the constants $\varepsilon, k$ are both positive. To study the homogeneous wave equation with such data, it is convenient to introduce the Banach space

$$
X=\left\{u(x, t): \partial_{x}^{\alpha} u(x, t) \in \mathcal{C}\left(\mathbb{R}^{3} \times \mathbb{R}\right) \quad \text { for }|\alpha| \leq 2, \quad\|u\|<\infty\right\} .
$$

Here, the norm $\|\cdot\|$ is defined by

$$
\|u\|=\sum_{|\alpha| \leq 2} \sup _{\substack{x \in \mathbb{R}^{3} \\ t \in \mathbb{R}}}\left|\partial_{x}^{\alpha} u(x, t)\right| \cdot W_{k}(|x|,|t|),
$$

where the weight function $W_{k}$ is of the form

$$
W_{k}(|x|,|t|)=\langle|t|+|x|\rangle^{\mu}\langle|t|-|x|\rangle^{\nu}\left(1+\ln \frac{\langle|t|+|x|\rangle}{\langle|t|-|x|\rangle}\right)^{-\delta_{1 k}}
$$

with $\mu=\min (k, 1), \nu=\max (k-1,0)$ and $\delta_{1 k}$ the usual Kronecker delta.

For the proof of the following lemma, we refer the reader to $[1,16]$.

Lemma 2.1. Suppose $\varphi \in \mathcal{C}^{3}\left(\mathbb{R}^{3}\right)$ and $\psi \in \mathcal{C}^{2}\left(\mathbb{R}^{3}\right)$ are subject to $(2.3)$ for some $\varepsilon, k>0$. Then the Cauchy problem (2.1)-(2.2) admits a unique solution $u_{0}^{-} \in X$ (2.4). Moreover, the estimate $\left\|u_{0}^{-}\right\| \leq C_{0} \varepsilon$ holds for some constant $C_{0}$ that depends solely on $k$.

Next, we turn to the nonlinear wave equation with potential

$$
\partial_{t}^{2} u-\Delta u+V(x) \cdot u=F(u) \quad \text { in } \mathbb{R}^{3} \times \mathbb{R} .
$$

When it comes to the potential term $V(x)$, we shall assume that

$$
\sum_{|\alpha| \leq 2}\left|\partial_{x}^{\alpha} V(x)\right| \leq V_{0}\langle x\rangle^{-l}, \quad l>2
$$

for some small $V_{0}>0$. When it comes to the nonlinear term $F(u)$, we require that

$$
F \in \mathcal{C}^{2}(\mathbb{R}) ; \quad F(0)=F^{\prime}(0)=F^{\prime \prime}(0)=0
$$

and that the estimate

$$
\left|F^{\prime \prime}(u)-F^{\prime \prime}(v)\right| \leq \begin{cases}A|u-v|^{p-2} & \text { if } 1+\sqrt{2}<p<3 \\ A(|u|+|v|)^{p-3}|u-v| & \text { if } p \geq 3\end{cases}
$$

holds for some $A>0$ and $p>1+\sqrt{2}$ whenever $|u|,|v| \leq 1$.

Recall that we seek a solution to the integral equation (1.9), where $u_{0}^{-}$is the solution of Lemma 2.1. One of our assumptions (1.3) ensures that $k \geq 2 /(p-1)$, where $k$ is the decay rate of the initial data. There is no loss of generality in decreasing the decay rate $k$, as long as the lower bound is not contradicted. Since we actually have

$$
\frac{2}{p-1}<\min (p-1,1+1 / p)
$$

whenever $p>1+\sqrt{2}$, this means there is no loss of generality in assuming

$$
\frac{2}{p-1} \leq k<\min (p-1,1+1 / p), \quad p>1+\sqrt{2} .
$$


Now, in the definition (2.6) of our weight function, we also introduced the parameters

$$
\mu=\min (k, 1), \quad \nu=\max (k-1,0) .
$$

Under our assumption (2.10), those are easily seen to satisfy the conditions

$$
0 \leq \nu \leq \nu p<1, \quad \mu+\nu=k, \quad \mu p \geq \min (k+2, p)>2 .
$$

Remark 2.2. In what follows, we shall frequently use the bracket notation $\langle s\rangle=1+|s|$.

For the proof of the following lemma, we refer the reader to $[1,16]$.

Lemma 2.3. Let $r, t>0$ be arbitrary. Assuming that $k>0$, one has

$$
\int_{|t-r|}^{t+r}\langle\gamma\rangle^{-k} d \gamma \leq C(k) \cdot r W_{k}(r, t)^{-1}
$$

where the weight function $W_{k}$ is given by (2.6).

Lemma 2.4. Let $w \geq 0$ be arbitrary. Given constants $a<1$ and $b \geq 0$, one has

$$
A_{b} \equiv \int_{0}^{w}\langle\gamma\rangle^{-a}\left(1+\ln \frac{\langle w\rangle}{\langle\gamma\rangle}\right)^{b} d \gamma \leq C(a, b) \cdot w\langle w\rangle^{-a}
$$

Proof. When $0 \leq w \leq 1$, the desired estimate is easy to obtain. Assume now that $w \geq 1$. Since the given integral is increasing in $b$, it suffices to treat the case that $b$ is an integer. Integrating by parts and using the fact that $a<1$, we find

$$
\begin{aligned}
A_{b} & =\left[\frac{\langle\gamma\rangle^{1-a}}{1-a} \cdot\left(1+\ln \frac{\langle w\rangle}{\langle\gamma\rangle}\right)^{b}\right]_{\gamma=0}^{w}+\int_{0}^{w} \frac{b\langle\gamma\rangle^{-a}}{1-a} \cdot\left(1+\ln \frac{\langle w\rangle}{\langle\gamma\rangle}\right)^{b-1} d \gamma \\
& \leq \frac{\langle w\rangle^{1-a}}{1-a}+\frac{b}{1-a} \cdot A_{b-1} .
\end{aligned}
$$

Since the desired (2.13) holds trivially when $b=0$, the result follows by induction.

Lemma 2.5. Let $\gamma \in \mathbb{R}$ and $z \geq|\gamma|$ be arbitrary. Suppose that (2.10), (2.11) hold. Given any constants $b \geq 0$ and $l>2$, one has

$$
I \equiv \int_{z}^{\infty}\langle\beta+\gamma\rangle^{1-l} \cdot\langle\beta\rangle^{-\mu}\left(1+\ln \frac{\langle\beta\rangle}{\langle\gamma\rangle}\right)^{b} d \beta \leq C\langle z\rangle^{-\mu}
$$

as well as

$$
J_{b} \equiv \int_{z}^{\infty}\langle\beta\rangle^{1-\mu p}\left(1+\ln \frac{\langle\beta\rangle}{\langle\gamma\rangle}\right)^{b} d \beta \leq C\langle z\rangle^{2-\mu p}\left(1+\ln \frac{\langle z\rangle}{\langle\gamma\rangle}\right)^{b} .
$$

Besides, the constant $C$ is independent of $\gamma$ and $z$.

Proof. To prove (2.14), it is convenient to divide the given integral into two parts. Let

$$
I_{1} \equiv \int_{\max (z, 2|\gamma|)}^{\infty}\langle\beta+\gamma\rangle^{1-l} \cdot\langle\beta\rangle^{-\mu}\left(1+\ln \frac{\langle\beta\rangle}{\langle\gamma\rangle}\right)^{b} d \beta
$$

Since $2(1+\beta+\gamma) \geq 1+\beta$ within the region of integration and since $l>2$, we find

$$
I_{1} \leq C_{1} \int_{z}^{\infty}\langle\beta\rangle^{1-l} \cdot\langle\beta\rangle^{-\mu} \cdot\langle\beta\rangle^{l-2} d \beta=C_{2}\langle z\rangle^{-\mu}
$$


because $\mu>0$ by (2.11). Thus, it remains to now treat

$$
I-I_{1}=\int_{z}^{\max (z, 2|\gamma|)}\langle\beta+\gamma\rangle^{1-l} \cdot\langle\beta\rangle^{-\mu}\left(1+\ln \frac{\langle\beta\rangle}{\langle\gamma\rangle}\right)^{b} d \beta .
$$

Since $|\gamma| \leq z \leq \beta \leq 2|\gamma|$ within the region of integration, we find

$$
I-I_{1} \leq C\langle z\rangle^{-\mu} \int_{z}^{\infty}\langle\beta+\gamma\rangle^{1-l} d \beta \leq C\langle z\rangle^{-\mu}
$$

because $l>2$. This proves our first assertion (2.14).

To prove (2.15), we modify our approach in the previous lemma as follows. Integrating by parts, we get

$$
\begin{aligned}
J_{b} & =\left[\frac{\langle\beta\rangle^{2-\mu p}}{2-\mu p} \cdot\left(1+\ln \frac{\langle\beta\rangle}{\langle\gamma\rangle}\right)^{b}\right]_{\beta=z}^{\infty}-\int_{z}^{\infty} \frac{b\langle\beta\rangle^{1-\mu p}}{2-\mu p} \cdot\left(1+\ln \frac{\langle\beta\rangle}{\langle\gamma\rangle}\right)^{b-1} d \beta \\
& =\frac{\langle z\rangle^{2-\mu p}}{\mu p-2} \cdot\left(1+\ln \frac{\langle z\rangle}{\langle\gamma\rangle}\right)^{b}+\frac{b}{\mu p-2} \cdot J_{b-1}
\end{aligned}
$$

since $\mu p>2$ by (2.12). Iterating this fact a sufficient number of times, we thus obtain

$$
J_{b} \leq C\langle z\rangle^{2-\mu p}\left(1+\ln \frac{\langle z\rangle}{\langle\gamma\rangle}\right)^{b}+C \int_{z}^{\infty}\langle\beta\rangle^{1-\mu p} \cdot\left(1+\ln \frac{\langle\beta\rangle}{\langle\gamma\rangle}\right)^{b^{\prime}} d \beta
$$

for some $b^{\prime} \leq 0$. Here, the logarithmic factor in the integrand is bounded since $\beta \geq z \geq|\gamma|$ within the region of integration. Recalling that $\mu p>2$, we may thus deduce (2.15).

Lemma 2.6. Let $r>0$ and $t \in \mathbb{R}$. Assuming (2.10), (2.11) and that $l>2$, one has

$$
\mathcal{I} \equiv \int_{-\infty}^{t} \int_{\left|\lambda_{-}\right|}^{\lambda_{+}}\langle\lambda\rangle^{1-l} \cdot W_{k}(\lambda,|\tau|)^{-1} d \lambda d \tau \leq C r \cdot W_{k}(r,|t|)^{-1}
$$

where $\lambda_{ \pm}=t-\tau \pm r, W_{k}$ is given by (2.6) and the constant $C$ is independent of $r, t$.

Proof. We divide the given integral into two parts.

First, we treat the part in which $\tau \geq 0$. For this part, we have to show that

$$
\mathcal{I}_{1} \equiv \int_{0}^{t} \int_{\left|\lambda_{-}\right|}^{\lambda_{+}}\langle\lambda\rangle^{1-l} \cdot W_{k}(\lambda, \tau)^{-1} d \lambda d \tau \leq C r \cdot W_{k}(r, t)^{-1}
$$

for each $t>0$. Recalling the definition (2.6) of $W_{k}$, let us then write

$$
\mathcal{I}_{1}=\int_{0}^{t} \int_{|t-\tau-r|}^{t-\tau+r}\langle\lambda\rangle^{1-l} \cdot\langle\lambda+\tau\rangle^{-\mu}\langle\lambda-\tau\rangle^{-\nu}\left(1+\ln \frac{\langle\lambda+\tau\rangle}{\langle\lambda-\tau\rangle}\right)^{\delta_{1 k}} d \lambda d \tau
$$

and change variables by $\beta=\lambda-\tau, \gamma=\lambda+\tau$ to arrive at

$$
\mathcal{I}_{1} \leq C \int_{|t-r|}^{t+r}\langle\gamma\rangle^{-\mu} \int_{-\gamma}^{\gamma}\langle\beta+\gamma\rangle^{1-l} \cdot\langle\beta\rangle^{-\nu}\left(1+\ln \frac{\langle\gamma\rangle}{\langle\beta\rangle}\right)^{\delta_{1 k}} d \beta d \gamma .
$$

According to Lemma 3.2 in [7], one does have the estimate

$$
\int_{-\gamma}^{\gamma}\langle\beta+\gamma\rangle^{1-l} \cdot\langle\beta\rangle^{-\nu}\left(1+\ln \frac{\langle\gamma\rangle}{\langle\beta\rangle}\right)^{\delta_{1 k}} d \beta \leq C\langle\gamma\rangle^{-\nu}
$$


whenever $l>2$ and $\nu<1$. In view of (2.12) then, the last two equations imply that

$$
\mathcal{I}_{1} \leq C \int_{|t-r|}^{t+r}\langle\gamma\rangle^{-\mu-\nu} d \gamma=C \int_{|t-r|}^{t+r}\langle\gamma\rangle^{-k} d \gamma
$$

Once we now invoke Lemma 2.3, we arrive at the desired estimate (2.16).

Next, we treat the part in which $\tau \leq 0$. For this part, we have to show that

$$
\mathcal{I}_{2} \equiv \int_{-\infty}^{\min (0, t)} \int_{\left|\lambda_{-}\right|}^{\lambda_{+}}\langle\lambda\rangle^{1-l} \cdot W_{k}(\lambda,-\tau)^{-1} d \lambda d \tau \leq C r \cdot W_{k}(r,|t|)^{-1}
$$

for any $t \in \mathbb{R}$ whatsoever. Recalling the definition (2.6) of $W_{k}$, let us then write

$$
\mathcal{I}_{2}=\int_{-\infty}^{\min (0, t)} \int_{\left|\lambda_{-}\right|}^{\lambda_{+}}\langle\lambda\rangle^{1-l} \cdot\langle\lambda-\tau\rangle^{-\mu}\langle\lambda+\tau\rangle^{-\nu}\left(1+\ln \frac{\langle\lambda-\tau\rangle}{\langle\lambda+\tau\rangle}\right)^{\delta_{1 k}} d \lambda d \tau .
$$

As $\tau \leq t$ within the region of integration, we have $\lambda \geq\left|\lambda_{-}\right| \geq t-\tau-r$. As $\tau \leq 0$, we also have $\bar{\lambda} \geq\left|\lambda_{-}\right| \geq|t-r|+\tau$. Changing variables by $\beta=\lambda-\tau$ and $\gamma=\lambda+\tau$, we then get

$$
\begin{aligned}
\mathcal{I}_{2} & \leq C \int_{t-r}^{t+r}\langle\gamma\rangle^{-\nu} \int_{\max (|\gamma|,|t-r|)}^{\infty}\langle\beta+\gamma\rangle^{1-l} \cdot\langle\beta\rangle^{-\mu}\left(1+\ln \frac{\langle\beta\rangle}{\langle\gamma\rangle}\right)^{\delta_{1 k}} d \beta d \gamma \\
& \leq C \int_{t-r}^{t+r}\langle\gamma\rangle^{-\nu} \cdot\langle z\rangle^{-\mu} d \gamma
\end{aligned}
$$

by Lemma 2.5 with $z=\max (|\gamma|,|t-r|)$. Note that this trivially implies

$$
\mathcal{I}_{2} \leq C \int_{t-r}^{t+r}\langle\gamma\rangle^{-\nu} \cdot\langle z\rangle^{-\mu} \cdot\left(1+\ln \frac{\langle z\rangle}{\langle\gamma\rangle}\right)^{b} d \gamma
$$

for any $b \geq 0$ and that we also have $\nu<1$ by (2.12). To establish the desired (2.17), we shall now show that the more general estimate

$$
\mathcal{I}_{2}^{\prime} \equiv \int_{t-r}^{t+r}\langle\gamma\rangle^{-c_{1}} \cdot\langle z\rangle^{-c_{2}} \cdot\left(1+\ln \frac{\langle z\rangle}{\langle\gamma\rangle}\right)^{b} d \gamma \leq C r \cdot W_{k}(r,|t|)^{-1}
$$

holds whenever $z=\max (|\gamma|,|t-r|), b \geq 0, c_{1}<1$ and $c_{1}+c_{2}=\mu+\nu=k$.

Case 1: When $t \geq 0$, we have $t-r \leq|t-r| \leq t+r$ and our definition (2.18) reads

$$
\mathcal{I}_{2}^{\prime}=\int_{|t-r|}^{t+r}\langle\gamma\rangle^{-k} d \gamma+\langle t-r\rangle^{-c_{2}} \int_{t-r}^{|t-r|}\langle\gamma\rangle^{-c_{1}} \cdot\left(1+\ln \frac{\langle t-r\rangle}{\langle\gamma\rangle}\right)^{b} d \gamma
$$

because $z=|\gamma|$ within the former integral and $z=|t-r|$ within the latter. This gives

$$
\mathcal{I}_{2}^{\prime} \leq C r \cdot W_{k}(r, t)^{-1}+\langle t-r\rangle^{-c_{2}} \int_{t-r}^{|t-r|}\langle\gamma\rangle^{-c_{1}} \cdot\left(1+\ln \frac{\langle t-r\rangle}{\langle\gamma\rangle}\right)^{b} d \gamma
$$

by Lemma 2.3. Since $c_{1}<1$ and since we may henceforth assume that $r \geq t$, we find

$$
\begin{aligned}
\mathcal{I}_{2}^{\prime} & \leq C r \cdot W_{k}(r, t)^{-1}+2\langle r-t\rangle^{-c_{2}} \int_{0}^{r-t}\langle\gamma\rangle^{-c_{1}} \cdot\left(1+\ln \frac{\langle r-t\rangle}{\langle\gamma\rangle}\right)^{b} d \gamma \\
& \leq C r \cdot W_{k}(r, t)^{-1}+C(r-t) \cdot\langle r-t\rangle^{-\mu-\nu}
\end{aligned}
$$

by Lemma 2.4 with $w=r-t \geq 0$. To deduce the desired (2.18), it remains to show that

$$
(r-t) \cdot\langle r-t\rangle^{-\mu-\nu} \leq C r \cdot\langle r+t\rangle^{-\mu}\langle r-t\rangle^{-\nu} \quad \text { when } r \geq t \geq 0 .
$$


If $r \geq t$ and $r \leq 1$, this is easy to see because $r-t \leq r$ and $\langle r-t\rangle$ is equivalent to $\langle r+t\rangle$. If $r \geq t$ and $r \geq 1$, on the other hand, $r$ is equivalent to $\langle r+t\rangle$ and we similarly get

$$
(r-t) \cdot\langle r-t\rangle^{-\mu-\nu} \leq\langle r+t\rangle^{1-\mu}\langle r-t\rangle^{-\nu} \leq C r \cdot\langle r+t\rangle^{-\mu}\langle r-t\rangle^{-\nu}
$$

because $\mu \leq 1$ by $(2.11)$.

Case 2: When $t \leq 0$, we have $|r+t| \leq r+|t|=r-t$, so our definition (2.18) reads

$$
\mathcal{I}_{2}^{\prime}=\langle r-t\rangle^{-c_{2}} \int_{t-r}^{t+r}\langle\gamma\rangle^{-c_{1}} \cdot\left(1+\ln \frac{\langle r-t\rangle}{\langle\gamma\rangle}\right)^{b} d \gamma .
$$

Subcase 2a: If it happens that $|t| \leq 3 r$, we proceed as in the previous case to obtain

$$
\mathcal{I}_{2}^{\prime} \leq\langle r-t\rangle^{-c_{2}} \int_{t-r}^{r-t}\langle\gamma\rangle^{-c_{1}} \cdot\left(1+\ln \frac{\langle r-t\rangle}{\langle\gamma\rangle}\right)^{b} d \gamma \leq C(r-t) \cdot\langle r-t\rangle^{-\mu-\nu}
$$

by Lemma 2.4. Since $r-t=r+|t| \leq 4 r$ for this subcase, the desired (2.18) follows.

Subcase 2b: If it happens that $-t=|t| \geq 3 r$, then $\langle r+t\rangle$ is equivalent to $\langle r-t\rangle$ because

$$
|r+t| \leq r+|t|=r-t \leq-2(r+t)
$$

for this subcase. In particular, equation (2.19) trivially leads to

$$
\mathcal{I}_{2}^{\prime} \leq C\langle r \pm t\rangle^{-\mu-\nu} \int_{t-r}^{t+r} d \gamma \leq C r\langle r \pm t\rangle^{-\mu-\nu}
$$

which implies the desired estimate (2.18) whenever $\langle r+t\rangle$ is equivalent to $\langle r-t\rangle$.

Lemma 2.7. Let $r>0$ and $t \in \mathbb{R}$. Assuming (2.10) and (2.11), one has

$$
\mathcal{J} \equiv \int_{-\infty}^{t} \int_{\left|\lambda_{-}\right|}^{\lambda_{+}} \lambda \cdot W_{k}(\lambda,|\tau|)^{-p} d \lambda d \tau \leq C r \cdot W_{k}(r,|t|)^{-1},
$$

where $\lambda_{ \pm}=t-\tau \pm r, W_{k}$ is given by (2.6) and the constant $C$ is independent of $r, t$.

Proof. We proceed as in the proof of the previous lemma.

First, we treat the part in which $\tau \geq 0$. For this part, we have to show that

$$
\mathcal{J}_{1} \equiv \int_{0}^{t} \int_{\left|\lambda_{-}\right|}^{\lambda_{+}} \lambda \cdot W_{k}(\lambda, \tau)^{-p} d \lambda d \tau \leq C r \cdot W_{k}(r, t)^{-1}
$$

for each $t>0$. Let us then change variables by $\beta=\lambda-\tau, \gamma=\lambda+\tau$ to get

$$
\mathcal{J}_{1} \leq C \int_{|t-r|}^{t+r}\langle\gamma\rangle^{-\mu p} \int_{-\gamma}^{\gamma}(\beta+\gamma) \cdot\langle\beta\rangle^{-\nu p}\left(1+\ln \frac{\langle\gamma\rangle}{\langle\beta\rangle}\right)^{p \delta_{1 k}} d \beta d \gamma .
$$

According to Lemma 3.3 in [7], one does have the estimate

$$
\int_{-\gamma}^{\gamma}(\beta+\gamma) \cdot\langle\beta\rangle^{-\nu p}\left(1+\ln \frac{\langle\gamma\rangle}{\langle\beta\rangle}\right)^{p \delta_{1 k}} d \beta \leq C\langle\gamma\rangle^{\mu p-k}
$$

whenever (2.10) and (2.11) hold. In particular, the last two equations also imply

$$
\mathcal{J}_{1} \leq C \int_{|t-r|}^{t+r}\langle\gamma\rangle^{-k} d \gamma \leq C r \cdot W_{k}(r, t)^{-1}
$$

by means of Lemma 2.3. This proves the desired estimate (2.20). 
Next, we treat the part in which $\tau \leq 0$. For this part, we have to show that

$$
\mathcal{J}_{2} \equiv \int_{-\infty}^{\min (0, t)} \int_{\left|\lambda_{-}\right|}^{\lambda_{+}} \lambda \cdot W_{k}(\lambda,-\tau)^{-p} d \lambda d \tau \leq C r \cdot W_{k}(r,|t|)^{-1}
$$

for any $t \in \mathbb{R}$ whatsoever. Recalling the definition (2.6) of $W_{k}$, let us then write

$$
\mathcal{J}_{2}=\int_{-\infty}^{\min (0, t)} \int_{\left|\lambda_{-}\right|}^{\lambda_{+}} \lambda \cdot\langle\lambda-\tau\rangle^{-\mu p}\langle\lambda+\tau\rangle^{-\nu p}\left(1+\ln \frac{\langle\lambda-\tau\rangle}{\langle\lambda+\tau\rangle}\right)^{p \delta_{1 k}} d \lambda d \tau .
$$

Changing variables by $\beta=\lambda-\tau$ and $\gamma=\lambda+\tau$, we find

$$
\begin{aligned}
\mathcal{J}_{2} & \leq C \int_{t-r}^{t+r}\langle\gamma\rangle^{-\nu p} \int_{\max (|\gamma|,|t-r|)}^{\infty}(\beta+\gamma) \cdot\langle\beta\rangle^{-\mu p}\left(1+\ln \frac{\langle\beta\rangle}{\langle\gamma\rangle}\right)^{p \delta_{1 k}} d \beta d \gamma \\
& \leq C \int_{t-r}^{t+r}\langle\gamma\rangle^{-\nu p} \int_{\max (|\gamma|,|t-r|)}^{\infty}\langle\beta\rangle^{1-\mu p}\left(1+\ln \frac{\langle\beta\rangle}{\langle\gamma\rangle}\right)^{p \delta_{1 k}} d \beta d \gamma
\end{aligned}
$$

because $\beta+\gamma \leq \beta+|\gamma| \leq 2 \beta$ within the region of integration. This already implies

$$
\mathcal{J}_{2} \leq C \int_{t-r}^{t+r}\langle\gamma\rangle^{-\nu p} \cdot\langle z\rangle^{2-\mu p} \cdot\left(1+\ln \frac{\langle z\rangle}{\langle\gamma\rangle}\right)^{p \delta_{1 k}} d \gamma
$$

by Lemma 2.5 with $z=\max (|\gamma|,|t-r|)$. Moreover, we also have

$$
2-\mu p=2-k p+\nu p \leq-k+\nu p
$$

because $\mu+\nu=k$ and $k \geq 2 /(p-1)$ by assumption. This allows us to conclude that

$$
\mathcal{J}_{2} \leq C \int_{t-r}^{t+r}\langle\gamma\rangle^{-\nu p} \cdot\langle z\rangle^{-(k-\nu p)} \cdot\left(1+\ln \frac{\langle z\rangle}{\langle\gamma\rangle}\right)^{p \delta_{1 k}} d \gamma
$$

As $\nu p<1$ by (2.12), we may then invoke our estimate (2.18) to complete the proof.

\section{Existence of the Scattering Operator}

In this section, we turn to the proofs of Theorem 1.1 and Theorem 1.3. Our first step is to establish the basic estimate for the existence proof.

Lemma 3.1. Suppose $V(x)$ satisfies (2.7) and $F(u)$ satisfies (2.8), (2.9). Assume that (2.10) and (2.11) also hold. Letting $\mathscr{L}$ be the Duhamel operator (1.10), one then has

$$
\|\mathscr{L} F(u)\| \leq C_{1}\|u\|^{p}, \quad\|\mathscr{L}(V u)\| \leq C_{1} V_{0}\|u\|
$$

for each $u \in X(2.4)$ with $\|u\| \leq 1$. Besides, the constant $C_{1}$ is independent of $u, V_{0}$.

Proof. Recall the definition (1.10) of the Duhamel operator, according to which

$$
[\mathscr{L} F(u)](x, t)=\frac{1}{4 \pi} \int_{-\infty}^{t}(t-\tau) \int_{|\xi|=1} F(u(x+(t-\tau) \xi, \tau)) d S_{\xi} d \tau .
$$

Using a direct differentiation, we then find

$$
\partial_{x}^{\alpha}[\mathscr{L} F(u)](x, t)=\frac{1}{4 \pi} \int_{-\infty}^{t} \frac{1}{t-\tau} \int_{|y-x|=t-\tau} \partial_{y}^{\alpha} F(u(y, \tau)) d S_{y} d \tau
$$

for each multi-index $\alpha$. 
To estimate the integrand, we use the fact that

$$
\left|\partial_{y}^{\alpha} F(u(y, \tau))\right| \leq\left. C|| u\right|^{p} \cdot W_{k}(|y|,|\tau|)^{-p}, \quad|\alpha| \leq 2 .
$$

One can easily obtain this fact using our assumptions (2.8), (2.9) on $F$ and the definition of our norm (2.5). Let us merely treat the case $|\alpha|=0$ here, as the cases $|\alpha|=1,2$ are quite similar. First, we combine (2.8) with (2.9) to find that

$$
|F(u)| \leq C|u|^{p} \quad \text { if }|u| \leq 1 \text {. }
$$

Next, we recall the definition (2.5) of our norm, according to which

$$
|u(y, \tau)| \leq\|u\| \cdot W_{k}(|y|,|\tau|)^{-1} .
$$

Since $\|u\| \leq 1$ by assumption and since $W_{k} \geq 1$ by definition (2.6), we do have $|u| \leq 1$ here. Once we now combine the last two equations, we obtain (3.3) for the case $|\alpha|=0$.

In order to proceed, we shall also need to invoke the following lemma from [13].

Lemma 3.2. Given $t>0, x \in \mathbb{R}^{3}$ and a continuous function $f: \mathbb{R} \rightarrow \mathbb{R}$, one has

$$
\frac{1}{t} \int_{|y-x|=t} f(|y|) d S_{y}=\frac{2 \pi}{r} \int_{|t-r|}^{t+r} \lambda f(\lambda) d \lambda
$$

where we have set $r=|x|$ for convenience.

Inserting our estimate (3.3) into (3.2) and using Lemma 3.2, we now find that

$$
\left|\partial_{x}^{\alpha} \mathscr{L} F(u)\right| \leq \frac{C \|\left. u\right|^{p}}{r} \int_{-\infty}^{t} \int_{|t-\tau-r|}^{t-\tau+r} \lambda \cdot W_{k}(\lambda,|\tau|)^{-p} d \lambda d \tau
$$

whenever $|\alpha| \leq 2$. In view of our estimate in Lemma 2.7 , this actually implies

$$
\left|\partial_{x}^{\alpha} \mathscr{L} F(u)\right| \leq C|| u||^{p} \cdot W_{k}(r,|t|)^{-1}, \quad|\alpha| \leq 2 .
$$

In view of the definition (2.5) of our norm, it thus implies our first assertion in (3.1).

To prove our second assertion in (3.1), we slightly modify our approach as follows. First, we return to $(3.2)$ and replace $F(u)$ by $V u$. To estimate the integrand, we now note that

$$
\left|\partial_{y}^{\alpha}[V(y) \cdot u(y, \tau)]\right| \leq C V_{0}|| u|| \cdot\langle y\rangle^{-l} W_{k}(|y|,|\tau|)^{-1}, \quad|\alpha| \leq 2
$$

because of (2.5) and (2.7). Using this fact and Lemma 3.2, we find that

$$
\left|\partial_{x}^{\alpha} \mathscr{L}(V u)\right| \leq \frac{C V_{0}\|u\|}{r} \int_{-\infty}^{t} \int_{|t-\tau-r|}^{t-\tau+r}\langle\lambda\rangle^{1-l} \cdot W_{k}(\lambda,|\tau|)^{-1} d \lambda d \tau
$$

whenever $|\alpha| \leq 2$. In view of Lemma 2.6 and (2.5), the desired estimate follows.

We are finally in a position to give the proofs of Theorem 1.1 and Theorem 1.3.

Proof of Theorem 1.1. Our iteration argument is almost identical with that of $[1,6]$, so we only give a sketch of the proof. As we have already mentioned earlier, one may decrease the decay rate $k$ of the initial data to ensure that (2.10) holds without loss of generality. We let $u_{0}=u_{0}^{-}$be the solution given by Lemma 2.1 and recursively define

$$
u_{i+1}=u_{0}^{-}+\mathscr{L} F\left(u_{i}\right)-\mathscr{L}\left(V u_{i}\right), \quad i \geq 0 .
$$

According to Lemma 2.1, we then have $u_{0} \in X$ (2.4) and we also have

$$
\left\|u_{0}\right\| \leq C_{0} \varepsilon \text {. }
$$


In order to proceed, we shall assume that $\varepsilon, V_{0}$ are so small that

$$
2 C_{1}\left(2 C_{0} \varepsilon\right)^{p-1}+2 C_{1} V_{0} \leq 1, \quad 2 C_{0} \varepsilon \leq 1
$$

when $C_{1}$ is the constant appearing in Lemma 3.1. Then we have

$$
2 C_{1}\left(2\left\|u_{0}\right\|\right)^{p-1}+2 C_{1} V_{0} \leq 1, \quad 2\left\|u_{0}\right\| \leq 1 .
$$

Using Lemma 3.1 and induction, we now find that $\left\|u_{i}\right\| \leq 2\left\|u_{0}\right\|$ for all $i$. In particular, the whole sequence $\left\{u_{i}\right\}$ lies in $X$. Using Lemma 3.1 and a contraction argument, as in $[1,6]$, we deduce the existence of a unique solution $u \in X$ to the integral equation (1.9).

Proof of Theorem 1.3. Our first step is to establish (1.13), which asserts that

$$
\left\|u-u_{0}^{-}\right\|_{e} \rightarrow 0 \quad \text { as } t \rightarrow-\infty .
$$

To prove this fact, as it is well-known, it suffices to obtain an estimate of the form

$$
\int_{-\infty}^{t}\|F(u)\|_{L^{2}\left(\mathbb{R}^{3}\right)} d \tau+\int_{-\infty}^{t}\|V u\|_{L^{2}\left(\mathbb{R}^{3}\right)} d \tau \leq C\langle t\rangle^{-\delta}, \quad t \leq 0
$$

for some $\delta>0$. Let us then focus on the derivation of (3.5), instead.

We recall that we are making two different kinds of assumptions in this theorem, as we can get better results in the absence of the potential. More precisely, we are assuming either

$$
k p \geq k+2, \quad k p>5 / 2, \quad V(x) \equiv 0
$$

or else

$$
k p \geq k+2, \quad k>1, \quad V(x) \not \equiv 0 .
$$

For the latter case, however, we are additionally assuming that $V(x)$ satisfies (2.7).

Case 1: Suppose (3.6) holds. To establish (3.5), it then suffices to show that

$$
G_{1}(\tau) \equiv \int_{\mathbb{R}^{3}} F(u(x, \tau))^{2} d x \leq C\langle\tau\rangle^{-2 \delta-2}, \quad \tau \leq 0
$$

for some $\delta>0$. Now, using our assumptions (2.8), (2.9) on $F$ and the definition (2.5) of our norm, one easily finds that

$$
F(u(x, \tau))^{2} \leq C|u(x, \tau)|^{2 p} \leq C|| u \|^{2 p} \cdot W_{k}(|x|,|\tau|)^{-2 p}
$$

because $u \in X$ by Theorem 1.1. Recall that the weight function (2.6) is given by

$$
W_{k}(|x|,|\tau|)=\langle|\tau|+|x|\rangle^{\mu}\langle|\tau|-|x|\rangle^{\nu}\left(1+\ln \frac{\langle|\tau|+|x|\rangle}{\langle|\tau|-|x|\rangle}\right)^{-\delta_{1 k}}
$$

where $\mu=\min (k, 1), \nu=k-\mu$ and $\delta_{1 k}$ is the usual Kronecker delta. Inserting the last two equations in our definition (3.8), we now switch to polar coordinates to find that

$$
G_{1}(\tau) \leq C \int_{0}^{\infty}\langle|\tau|+r\rangle^{2-2 \mu p}\langle|\tau|-r\rangle^{-2 \nu p}\left(1+\ln \frac{\langle|\tau|+r\rangle}{\langle|\tau|-r\rangle}\right)^{2 p \delta_{1 k}} d r .
$$


Note that each of $\langle|\tau| \pm r\rangle$ is equivalent to $\langle r\rangle$ whenever $r \geq 2|\tau|$, while each of $\langle|\tau| \pm r\rangle$ is equivalent to $\langle\tau\rangle$ whenever $|\tau| \geq 2 r$. Thus, the last equation also implies

$$
\begin{aligned}
G_{1}(\tau) \leq & C \int_{2|\tau|}^{\infty}\langle r\rangle^{2-2(\mu+\nu) p} d r+C\langle\tau\rangle^{2-2(\mu+\nu) p} \int_{0}^{|\tau| / 2} d r \\
& +C \int_{|\tau| / 2}^{2|\tau|}\langle|\tau|+r\rangle^{2-2 \mu p}\langle|\tau|-r\rangle^{-2 \nu p}\left(1+\ln \frac{\langle|\tau|+r\rangle}{\langle|\tau|-r\rangle}\right)^{2 p \delta_{1 k}} d r
\end{aligned}
$$

Here, $\mu+\nu=k$ by definition (2.11), so we actually have

$$
2-2(\mu+\nu) p=2-2 k p \leq-2 k-2<-2
$$

because $2 k p \geq 2 k+4$ by (3.6). Combining the last two equations, we then get

$$
G_{1}(\tau) \leq C\langle\tau\rangle^{3-2 k p}+C\langle\tau\rangle^{2-2 \mu p+\delta} \int_{|\tau| / 2}^{2|\tau|}\langle|\tau|-r\rangle^{-2 \nu p} d r
$$

for any $\delta>0$. Note that this trivially implies

$$
\begin{aligned}
G_{1}(\tau) & \leq C\langle\tau\rangle^{3-2 k p}+C\langle\tau\rangle^{2-2 \mu p+\delta}+C\langle\tau\rangle^{3-2(\mu+\nu) p+2 \delta} \\
& \leq C\langle\tau\rangle^{3-2 k p+2 \delta}+C\langle\tau\rangle^{2-2 \mu p+2 \delta}
\end{aligned}
$$

because $\mu+\nu=k$ by above. Now, our assumption (3.6) ensures that

$$
k p \geq k+2, \quad k p>5 / 2 .
$$

In view of our definition (2.11), this also gives

$$
\mu p=\min (k p, p) \geq \min (k+2, p)>2
$$

because $k>0$ and $p>1+\sqrt{2}$. In particular, we can always choose some $\delta$ such that

$$
0<2 \delta \leq \min (k p-5 / 2, \mu p-2) .
$$

For this choice of $\delta$, it is now easy to check that (3.10) implies the desired (3.8).

Case 2: Suppose (3.7) holds. Then $k p \geq k+2>3$, so the estimates of the previous case are still applicable. Thus, to establish (3.5), we need only worry about the potential term now. As in the previous case, it suffices to show that

$$
G_{2}(\tau) \equiv \int_{\mathbb{R}^{3}} V(x)^{2} \cdot u(x, \tau)^{2} d x \leq C\langle\tau\rangle^{-2 \varepsilon-2}, \quad \tau \leq 0
$$

for some $\varepsilon>0$. Arguing as before and using our assumption (2.7), we find

$$
G_{2}(\tau) \leq C \int_{0}^{\infty}\langle r\rangle^{2-2 l}\langle|\tau|+r\rangle^{-2 \mu}\langle|\tau|-r\rangle^{-2 \nu}\left(1+\ln \frac{\langle|\tau|+r\rangle}{\langle|\tau|-r\rangle}\right)^{2 \delta_{1 k}} d r
$$

In analogy with (3.9), we divide this integral into three parts, and we now get

$$
\begin{aligned}
G_{2}(\tau) \leq & C \int_{2|\tau|}^{\infty}\langle r\rangle^{2-2 l-2 k} d r+C\langle\tau\rangle^{-2 k} \int_{0}^{|\tau| / 2}\langle r\rangle^{2-2 l} d r \\
& +C\langle\tau\rangle^{2-2 l-2 \mu} \int_{|\tau| / 2}^{2|\tau|}\langle|\tau|-r\rangle^{-2 \nu}\left(1+\ln \frac{\langle|\tau|+r\rangle}{\langle|\tau|-r\rangle}\right)^{2 \delta_{1 k}} d r
\end{aligned}
$$


because $\mu+\nu=k$. Since $l>2$ by our assumption (2.7), we then get

$$
G_{2}(\tau) \leq C\langle\tau\rangle^{3-2 l-2 k}+C\langle\tau\rangle^{-2 k}+C\langle\tau\rangle^{2-2 l-2 \mu+\varepsilon} \int_{|\tau| / 2}^{2|\tau|}\langle|\tau|-r\rangle^{-2 \nu} d r
$$

for any $\varepsilon>0$. Note that this trivially implies

$$
\begin{aligned}
G_{2}(\tau) & \leq C\langle\tau\rangle^{-2 k}+C\langle\tau\rangle^{2-2 l-2 \mu+\varepsilon}+C\langle\tau\rangle^{3-2 l-2 k+2 \varepsilon} \\
& \leq C\langle\tau\rangle^{-2 k+2 \varepsilon}+C\langle\tau\rangle^{2-2 l-2 \mu+2 \varepsilon}
\end{aligned}
$$

because $l>2$. Now, our assumption (3.7) ensures that $k>1$, hence

$$
\mu=\min (k, 1)=1
$$

by our definition (2.11). In particular, we can always choose some $\varepsilon$ such that

$$
0<2 \varepsilon \leq \min (k-1, \mu+l-2) .
$$

For this choice of $\varepsilon$, it is now easy to check that (3.12) implies the desired (3.11).

This finally completes the proof of (3.5), which also implies our first assertion (1.13). To prove the remaining assertions of the theorem, we set

$$
\begin{aligned}
u_{0}^{+}(x, t)= & u(x, t)-\frac{1}{4 \pi} \int_{t}^{\infty} \frac{1}{\tau-t} \int_{|y-x|=\tau-t} F(u(y, \tau)) d S_{y} d \tau \\
& +\frac{1}{4 \pi} \int_{t}^{\infty} \frac{1}{\tau-t} \int_{|y-x|=\tau-t} V(y) \cdot u(y, \tau) d S_{y} d \tau
\end{aligned}
$$

As one can readily check, $u_{0}^{+}$is then a $\mathcal{C}^{2}$-solution to the homogeneous wave equation (1.8). Besides, the expression $u-u_{0}^{+}$bears a close resemblance to the Duhamel operator (1.10), so one may establish the convergence

$$
\left\|u-u_{0}^{+}\right\|_{e} \rightarrow 0 \quad \text { as } t \rightarrow+\infty
$$

in the exact same way that we obtained (1.13). Given some other $\mathcal{C}^{2}$-solution with the same properties as $u_{0}^{+}$, the difference $w$ of the two must satisfy the homogeneous equation (1.8) and its energy norm $\|w\|_{e}$ must tend to zero as $t \rightarrow+\infty$. Since this implies that $w \equiv 0$, the uniqueness assertion of the theorem follows as well.

\section{NON-EXISTENCE OF THE SCATTERING OPERATOR}

In this section, we give the proof of Theorem 1.6. One of the key ingredients in the proof is a standard fact about the wave equation. We only include its derivation here for the sake of completeness.

Lemma 4.1. Let the assumptions of Theorem 1.1 hold. Also, assume

$$
\varphi(x) \equiv 0, \quad \psi(x) \leq 0, \quad V(x) \leq 0 \quad \text { for all } x \in \mathbb{R}^{3} .
$$

Denote by $u$ and $u_{0}^{-}$the solutions of Theorem 1.1 and Lemma 2.1, respectively. Then

$$
u(x, t) \geq u_{0}^{-}(x, t)=\frac{|t|}{4 \pi} \int_{|\xi|=1}|\psi(x+t \xi)| d S_{\xi}
$$

for all $x \in \mathbb{R}^{3}$ and each $t \leq 0$. 
Proof. Repeating the iteration argument in the proof of Theorem 1.1, we let $u_{0}=u_{0}^{-}$and then recursively define a sequence of functions $\left\{u_{i}\right\}$ by setting

$$
u_{i+1}=u_{0}^{-}+\mathscr{L} F\left(u_{i}\right)-\mathscr{L}\left(V u_{i}\right), \quad i \geq 0 .
$$

Due to our assumption that $\varphi \equiv 0$, we have the explicit representation

$$
u_{0}(x, t)=u_{0}^{-}(x, t)=\frac{t}{4 \pi} \int_{|\xi|=1} \psi(x+t \xi) d S_{\xi} .
$$

Due to our assumption that $\psi \leq 0$, we thus have $u_{0}=u_{0}^{-} \geq 0$ for each $t \leq 0$.

Suppose $u_{i} \geq u_{0}^{-} \geq 0$ for some $i$ and each $t \leq 0$. When it comes to the expression

$$
\left[\mathscr{L} F\left(u_{i}\right)\right](x, t)=\frac{1}{4 \pi} \int_{-\infty}^{t} \frac{1}{t-\tau} \int_{|y-x|=t-\tau} F\left(u_{i}(y, \tau)\right) d S_{y} d \tau
$$

we defined in (1.10), we then have $F\left(u_{i}(y, \tau)\right)=u_{i}(y, \tau)^{p}$ whenever $t \leq 0$. Thus, the whole expression is non-negative for each $t \leq 0$. The same is true for $-\mathscr{L}\left(V u_{i}\right)$ because $V \leq 0$ by assumption. Using (4.2) and induction, we thus find $u_{i} \geq u_{0}^{-} \geq 0$ for all $i$ and each $t \leq 0$. Since the solution $u$ arises as the limit of the sequence $\left\{u_{i}\right\}$, the result follows.

We are now ready to give the proof of Theorem 1.6. We divide the proof into two parts, as we are making two different kinds of assumptions in this theorem.

Proof of Theorem 1.6 assuming (1.15). Our goal is to show that the unique solution of Theorem 1.1 does not exhibit the asymptotic behavior

$$
\left\|u-u_{0}^{-}\right\|_{e} \rightarrow 0 \quad \text { as } t \rightarrow-\infty .
$$

When it comes to this theorem, we are assuming the initial data are such that

$$
\varphi(x) \equiv 0, \quad \psi(x) \leq 0, \quad|\psi(x)| \geq \varepsilon_{1}\langle x\rangle^{-k-1}
$$

for some $\varepsilon_{1}>0$. In this case, we also assume that (1.15) holds, i.e., we assume that

$$
k p \geq k+2, \quad 0<k \leq 1, \quad 0 \not \equiv(x) \leq 0 .
$$

With $R>0$ to be specified below, let us fix a smooth test function $\zeta \geq 0$ such that

$$
\begin{aligned}
\zeta(x) & =1 & & \text { if }|x| \leq R ; \\
|\Delta \zeta(x)| & \leq\langle x\rangle^{-4} & & \text { if }|x| \geq R ; \\
|\zeta(x)|+|\nabla \zeta(x)| & =O\left(|x|^{-4}\right) & & \text { as }|x| \rightarrow \infty .
\end{aligned}
$$

We remark that our test function $\zeta$ lies in $L^{2}\left(\mathbb{R}^{3}\right)$ because of the last equation.

Suppose now, for the sake of contradiction, that (4.3) does hold. Since $\zeta \in L^{2}$ by above, we may then use Hölder's inequality to see that

$$
H_{1}(t)=\int_{\mathbb{R}^{3}} \zeta(x) \cdot \partial_{t}\left(u-u_{0}^{-}\right) d x
$$

is absolutely convergent on $\left(-\infty,-t_{0}\right)$ for some $t_{0}>0$ and that

$$
H_{1}(t) \rightarrow 0 \quad \text { as } t \rightarrow-\infty .
$$

By definition, the functions $u, u_{0}^{-}$are solutions to the equations

$$
\partial_{t}^{2} u=\Delta u+F(u)-V(x) \cdot u, \quad \partial_{t}^{2} u_{0}^{-}=\Delta u_{0}^{-} .
$$


Differentiating (4.9) under the integral sign, as we may, we thus obtain the identity

$$
H_{1}^{\prime}(t)=\int_{\mathbb{R}^{3}} \zeta \cdot \Delta\left(u-u_{0}^{-}\right) d x+\int_{\mathbb{R}^{3}} \zeta F(u) d x-\int_{\mathbb{R}^{3}} \zeta V u d x
$$

for all times $t<-t_{0}$.

To handle the first integral, we use the estimates of Theorem 1.1 and Lemma 2.1. Since both $u$ and $u_{0}^{-}$are known to lie in the Banach space (2.4), we certainly have

$$
\sum_{|\alpha| \leq 2}\left|\partial_{x}^{\alpha}\left[u(x, t)-u_{0}^{-}(x, t)\right]\right| \leq C W_{k}(|x|,|t|)^{-1},
$$

where $W_{k}$ is our weight function (2.6). We remark that the last equation reads

$$
\sum_{|\alpha| \leq 2}\left|\partial_{x}^{\alpha}\left[u(x, t)-u_{0}^{-}(x, t)\right]\right| \leq C\langle|x|+|t|\rangle^{-k}\left(1+\ln \frac{\langle|x|+|t|\rangle}{\langle|x|-|t|\rangle}\right)^{\delta_{1 k}}
$$

in this case, as $k \leq 1$ by our assumption (4.5). In particular, we trivially get

$$
\sum_{|\alpha| \leq 2}\left|\partial_{x}^{\alpha}\left[u(x, t)-u_{0}^{-}(x, t)\right]\right| \leq C\langle|x|+|t|\rangle^{-k} \cdot\langle x\rangle^{1 / 2}
$$

because the logarithmic factor is bounded whenever $|t| \geq 2|x|$.

Let us now return to our identity (4.11) and integrate by parts to obtain

$$
\begin{aligned}
H_{1}^{\prime}(t) & =\int_{\mathbb{R}^{3}} \Delta \zeta \cdot\left(u-u_{0}^{-}\right) d x+\int_{\mathbb{R}^{3}} \zeta F(u) d x-\int_{\mathbb{R}^{3}} \zeta V u d x \\
& \equiv A_{1}+A_{2}+A_{3} .
\end{aligned}
$$

One can easily justify this integration by parts using the decay properties (4.8) of $\zeta$ and our estimate (4.12). Next, we recall (4.6), according to which $\Delta \zeta=0$ whenever $|x| \leq R$. Once we now employ (4.7) and (4.12) to estimate the first integral, we find

$$
\left|A_{1}\right| \leq \int_{|x| \geq R}\langle x\rangle^{-4} \cdot C\langle t\rangle^{-k}\langle x\rangle^{1 / 2} d x \leq C\langle t\rangle^{-k}\langle R\rangle^{-1 / 2} .
$$

Inserting this estimate in (4.13), we thus find

$$
H_{1}^{\prime}(t) \geq \int_{\mathbb{R}^{3}} \zeta F(u) d x-\int_{\mathbb{R}^{3}} \zeta V u d x-C\langle t\rangle^{-k}\langle R\rangle^{-1 / 2}
$$

for all times $t<-t_{0}$ and some constant $C$ which is independent of $R$.

To handle the last two integrals, we recall the various sign conditions we have imposed. First of all, the test function $\zeta$ was chosen to be non-negative, while $V \leq 0$ by (4.5). Due to our choice (4.4) of initial data, we can also invoke Lemma 4.1 to get

$$
u(x, t) \geq \frac{|t|}{4 \pi} \int_{|\xi|=1}|\psi(x+t \xi)| d S_{\xi} \geq C|t| \int_{|\xi|=1}\langle x+t \xi\rangle^{-k-1} d S_{\xi} .
$$

This shows that $u$ is non-negative, so the same is true for $F(u)=u^{p}$ as well. Ignoring the first integral in (4.14) and using the fact that $\zeta V u \leq 0$, we then trivially get

$$
H_{1}^{\prime}(t) \geq \int_{|x| \leq|t| / 2}|\zeta V u| d x-C\langle t\rangle^{-k}\langle R\rangle^{-1 / 2}
$$

for all times $t<-t_{0}$ and some constant $C$ which is independent of $R$. 
When it comes to the integral in (4.16), we have $|t| \geq 2|x|$ within the region of integration. Assuming that $|t| \geq 1$ as well, we may now use (4.15) to find that

$$
u(x, t) \geq C|t| \int_{|\xi|=1}\langle x+t \xi\rangle^{-k-1} d S_{\xi} \geq C_{1}|t|^{-k}
$$

within the region of integration. In particular, (4.16) also implies

$$
H_{1}^{\prime}(t) \geq C_{1}|t|^{-k} \cdot\left[\int_{|x| \leq|t| / 2}|\zeta V| d x-C_{2}\langle R\rangle^{-1 / 2}\right]
$$

for all times $t<-t_{0}-1$, so it actually implies

$$
H_{1}^{\prime}(t) \geq C_{1}|t|^{-k} \cdot\left[\int_{|x| \leq R}|\zeta V| d x-C_{2}\langle R\rangle^{-1 / 2}\right]
$$

for all times $t<-t_{0}-2 R-1$. Besides, the constants $C_{1}, C_{2}$ are independent of $R$.

Since $\zeta(x)=1$ whenever $|x| \leq R$ by (4.6), the expression in brackets is given by

$$
\int_{|x| \leq R}|\zeta V| d x-C_{2}\langle R\rangle^{-1 / 2}=\int_{|x| \leq R}|V| d x-C_{2}\langle R\rangle^{-1 / 2} .
$$

Recalling (4.5), we now fix some $R_{0}>0$ so that $V \not \equiv 0$ in the ball $|x| \leq R_{0}$. Since

$$
\int_{|x| \leq R}|V| d x-C_{2}\langle R\rangle^{-1 / 2} \geq \int_{|x| \leq R_{0}}|V| d x-C_{2}\langle R\rangle^{-1 / 2}
$$

for each $R \geq R_{0}$, we then have

$$
\int_{|x| \leq R}|\zeta V| d x-C_{2}\langle R\rangle^{-1 / 2} \geq \frac{1}{2} \int_{|x| \leq R_{0}}|V| d x
$$

for a sufficiently large choice of $R$.

Fixing $R$ and inserting the last inequality in (4.17), we find

$$
H_{1}^{\prime}(t) \geq C_{3}|t|^{-k}
$$

for all times $t<-t_{1}$, where $t_{1}=t_{0}+2 R+1$. Since $k \leq 1$ by (4.5), this also gives

$$
H_{1}^{\prime}(t) \geq C_{3}|t|^{-k} \geq C_{3}|t|^{-1}
$$

and we may now integrate to get

$$
H_{1}(t)-H_{1}(2 t) \geq C_{3} \ln 2
$$

for all times $t<-t_{1}$. Note that this is contrary to (4.10). Since we obtained (4.10) under the assumption that (4.3) holds, the contradiction above disproves (4.3), as needed.

Proof of Theorem 1.6 assuming (1.16). Once again, we consider initial data with

$$
\varphi(x) \equiv 0, \quad \psi(x) \leq 0, \quad|\psi(x)| \geq \varepsilon_{1}\langle x\rangle^{-k-1}
$$

for some $\varepsilon_{1}>0$ and our goal is to disprove the convergence

$$
\left\|u-u_{0}^{-}\right\|_{e} \rightarrow 0 \quad \text { as } t \rightarrow-\infty .
$$

In this case, however, we assume that (1.16) holds, i.e., we assume that

$$
0<k, \quad k+2 \leq k p \leq 5 / 2, \quad V(x) \equiv 0 .
$$

Since $2 k p \geq 2 k+4>4$, it is clear that $\langle|x|+|t|\rangle^{-k p} \in L^{2}\left(\mathbb{R}^{3}\right)$ for all times. 
Suppose now, for the sake of contradiction, that (4.19) actually holds. Then

$$
H_{2}(t)=\int_{\mathbb{R}^{3}}\langle|x|+|t|\rangle^{-k p} \cdot \partial_{t}\left(u-u_{0}^{-}\right) d x
$$

is well-defined on $\left(-\infty,-T_{0}\right)$ for some $T_{0}>0$, and one easily finds that

$$
\begin{aligned}
H_{2}^{\prime}(t)= & k p \int_{\mathbb{R}^{3}}\langle|x|+|t|\rangle^{-k p-1} \cdot \partial_{t}\left(u-u_{0}^{-}\right) d x \\
& +\int_{\mathbb{R}^{3}}\langle|x|+|t|\rangle^{-k p} \cdot \Delta\left(u-u_{0}^{-}\right) d x \\
& +\int_{\mathbb{R}^{3}}\langle|x|+|t|\rangle^{-k p} \cdot F(u) d x \\
\equiv & B_{1}+B_{2}+B_{3}
\end{aligned}
$$

for all times $t<-T_{0}$.

First, we focus on $B_{1}$. According to Hölder's inequality, we have

$$
\left|B_{1}\right| \leq C|| u-u_{0}^{-} \|_{e} \cdot\left(\int_{\mathbb{R}^{3}}\langle|x|+|t|\rangle^{-2 k p-2} d x\right)^{1 / 2}
$$

and since $2 k p+2>6$ by above, this also implies that

$$
\left|B_{1}\right| \leq C|| u-u_{0}^{-} \|_{e} \cdot\langle t\rangle^{1 / 2-k p} .
$$

Next, we obtain the exact same estimate for $B_{2}$. Integrating by parts, we get

$$
\left|B_{2}\right| \leq C \int_{\mathbb{R}^{3}}\langle|x|+|t|\rangle^{-k p-1} \cdot\left|\nabla\left(u-u_{0}^{-}\right)\right| d x
$$

and then an application of Hölder's inequality gives

$$
\begin{aligned}
\left|B_{2}\right| & \leq C\left\|u-u_{0}^{-}\right\|_{e} \cdot\left(\int_{\mathbb{R}^{3}}\langle|x|+|t|\rangle^{-2 k p-2} d x\right)^{1 / 2} \\
& \leq C\left\|u-u_{0}^{-}\right\|_{e} \cdot\langle t\rangle^{1 / 2-k p}
\end{aligned}
$$

exactly as before.

Finally, we obtain a lower bound for $B_{3}$. Note that Lemma 4.1 applies to give

$$
u(x, t) \geq C|t| \int_{|\xi|=1}\langle x+t \xi\rangle^{-k-1} d S_{\xi}
$$

within the region of integration for all times $t<-T_{0}$. Since this implies

$$
u(x, t) \geq C|t| \int_{|\xi|=1}\langle x+t \xi\rangle^{-k-1} d S_{\xi} \geq C\langle t\rangle^{-k}
$$

whenever $|t| \geq 1$ and $|t| \geq 2|x|$, we then easily find that

$$
B_{3} \geq \int_{|t| / 3 \leq|x| \leq|t| / 2}\langle|x|+|t|\rangle^{-k p} \cdot u(x, t)^{p} d x \geq C\langle t\rangle^{3-2 k p}
$$

for all times $t<-T_{0}-1$.

Let us now return to (4.22). Using our estimates (4.23), (4.24) and (4.25), we get

$$
H_{2}^{\prime}(t) \geq C_{1}\langle t\rangle^{3-2 k p}-C_{2}|| u-u_{0}^{-} \|_{e} \cdot\langle t\rangle^{1 / 2-k p}
$$


for all times $t<-T_{0}-1$. Moreover, our assumption (4.20) ensures that

$$
\langle t\rangle^{1 / 2-k p} \leq\langle t\rangle^{3-2 k p}
$$

because it ensures that $k p \leq 5 / 2$. Combining the last two equations, we then find

$$
H_{2}^{\prime}(t) \geq\left[C_{1}-C_{2}\left\|u-u_{0}^{-}\right\|_{e}\right] \cdot\langle t\rangle^{3-2 k p}
$$

for all times $t<-T_{0}-1$. In view of (4.19), this also implies

$$
H_{2}^{\prime}(t) \geq C_{3}\langle t\rangle^{3-2 k p}
$$

for all sufficiently negative times, say, for all times $t<-T_{1}$.

Next, we integrate the last inequality to get

$$
H_{2}(t)-H_{2}(2 t) \geq C_{4}\langle t\rangle^{4-2 k p}
$$

for all times $t<-T_{1}$. Recalling our definition (4.21), we also have

$$
\left|H_{2}(s)\right| \leq\left\|\partial_{t}\left(u-u_{0}^{-}\right)\right\|_{L^{2}\left(\mathbb{R}^{3}\right)} \cdot\left(\int_{\mathbb{R}^{3}}\langle|x|+|s|\rangle^{-2 k p} d x\right)^{1 / 2}
$$

for any $s$ whatsoever. Since $2 k p \geq 2 k+4>4$ by (4.20), this actually implies

$$
\left|H_{2}(s)\right| \leq C|| u-u_{0}^{-} \|_{e}(s) \cdot\langle s\rangle^{3 / 2-k p}
$$

for any $s$ whatsoever. Once we now combine this fact with (4.26), we find

$$
\begin{aligned}
C_{4}\langle t\rangle^{4-2 k p} & \leq \sup _{2 t \leq s \leq t} 2\left|H_{2}(s)\right| \\
& \leq C\langle t\rangle^{3 / 2-k p} \cdot \sup _{2 t \leq s \leq t}\left\|u-u_{0}^{-}\right\|_{e}(s)
\end{aligned}
$$

for all times $t<-T_{1}$. Since $k p \leq 5 / 2$ by our assumption (4.20), this also gives

$$
\sup _{2 t \leq s \leq t}\left\|u-u_{0}^{-}\right\|_{e}(s) \geq C_{5}\langle t\rangle^{5 / 2-k p} \geq C_{5}
$$

contrary to (4.19). Thus, the convergence (4.19) cannot possibly hold, as needed.

\section{REFERENCES}

[1] F. AsAKURA, Existence of a global solution to a semilinear wave equation with slowly decreasing initial data in three space dimensions, Comm. Partial Differential Equations, 11 (1986), pp. 1459-1487.

[2] V. Georgiev, C. Heiming, And H. Kubo, Supercritical semilinear wave equation with non-negative potential, Comm. Partial Differential Equations, 26 (2001), pp. 2267-2303.

[3] R. T. GLASSEy, On the asymptotic behavior of nonlinear wave equations, Trans. Amer. Math. Soc., 182 (1973), pp. 187-200.

[4] _ Finite-time blow-up for solutions of nonlinear wave equations, Math. Z., 177 (1981), pp. 323-340.

[5] K. Hidano, Scattering problem for the nonlinear wave equation in the finite energy and conformal charge space, J. Funct. Anal., 187 (2001), pp. 274-307.

[6] F. John, Blow-up of solutions of nonlinear wave equations in three space dimensions, Manuscripta Math., 28 (1979), pp. 235-268.

[7] P. Karageorgis, Existence and blow up of small-amplitude nonlinear waves with a sign-changing potential. To appear in J. Differential Equations.

[8] — Small-data scattering for nonlinear waves with potential and initial data of critical decay. Preprint, 2004.

[9] K. KuBota, Existence of a global solution to a semi-linear wave equation with initial data of noncompact support in low space dimensions, Hokkaido Math. J., 22 (1993), pp. 123-180. 
[10] H. A. LEvine, Instability and nonexistence of global solutions to nonlinear wave equations of the form $P u_{t t}=-A u+\mathcal{F}(u)$, Trans. Amer. Math. Soc., 192 (1974), pp. 1-21.

[11] H. LindBlad AND C. D. SogGe, Long-time existence for small amplitude semilinear wave equations, Amer. J. Math., 118 (1996), pp. 1047-1135.

[12] A. Matsumura, On the asymptotic behavior of solutions of semilinear wave equations, Publ. Res. Inst. Math. Sci., 12 (1976/77), pp. 169-189.

[13] H. PECHER, Scattering for semilinear wave equations with small data in three space dimensions, Math. Z., 198 (1988), pp. 277-289.

[14] J. Schaeffer, The equation $u_{t t}-\Delta u=|u|^{p}$ for the critical value of $p$, Proc. Roy. Soc. Edinburgh Sect. A, 101 (1985), pp. 31-44.

[15] W. A. Strauss And K. Tsutaya, Existence and blow up of small amplitude nonlinear waves with a negative potential, Discrete Contin. Dynam. Systems, 3 (1997), pp. 175-188.

[16] K. Tsutaya, Global existence and the life span of solutions of semilinear wave equations with data of noncompact support in three space dimensions, Funkcial. Ekvac., 37 (1994), pp. 1-18.

[17] B. Yordanov And Q. S. Zhang, Finite-time blow up for wave equations with a potential, SIAM J. Math. Anal., 36 (2005), pp. 1426-1433.

E-mail address: pete@maths.tcd.ie

E-mail address: tsutaya@math.sci.hokudai.ac.jp

School of Mathematics, Trinity College, Dublin 2, Ireland.

Department of Mathematics, Hokkaido University, Sapporo 060-0810, Japan. 